\title{
E-Zigaretten: Ein vielschichtiges moralisches Dilemma
}

\author{
Constantine I. Vardavas ${ }^{a, b} \quad$ Israel T. Agaku ${ }^{a}$ \\ ${ }^{a}$ Center for Global Tobacco Control, Department of Social and Behavioral Sciences, Harvard School of Public Health, Boston, Mass., USA; \\ ${ }^{b}$ Clinic of Social and Family Medicine, University of Crete, Crete, Greece
}

\section{Zusammenfassung}

Elektrische Zigaretten (E-Zigaretten) sind neben anderen nikotinhaltigen Produkten, die weitgehend seit den späten 2000er Jahren im Handel sind, die neueste Stufe in der Entwicklung der Tabakkonsumgewohnheiten und werden in der internationalen Fachwelt des Gesundheitswesens vielfach kontrovers diskutiert. Dieser Beitrag ist der Versuch einer ganzheitlichen Betrachtung verschiedener von Wissenschaft und Politik aufgeworfener Fragen zur Gestaltung, Herstellung, Vermarktung und Nutzung von E-Zigaretten und den daraus folgenden Auswirkungen auf tabakbezogene Outcomes für den Einzelnen und die Gesellschaft, einschließlich der potenziellen Gesundheitsgefährdung. Ein alternatives neues Modell der Tabakepidemie mit drei Stadien wird vorgeschlagen, deren charakteristische Merkmale die Art der vorwiegend verwendeten Tabakprodukte und der Stand in Bezug zur globalen Tabakepidemie sind. Stadium 1 - vorwiegender Konsum konventioneller Tabakwaren in Ländern in der frühen Phase des Tabakkonsums (verschiedene Länder in Südostasien, China, Länder des Nahen Ostens sowie die afrikanische Region); Stadium 2 - Nebeneinander von konventionellen Tabakerzeugnissen und solchen mit modifiziertem Risiko in Ländern, in denen zunehmend Initiativen zur Tabakkontrolle ergriffen werden, die epidemiebedingte Mortalität bei Männern und Frauen jedoch noch an ihrem Höhepunkt ist (Länder in Süd- und Osteuropa, teilweise Asien und Südamerika); und Stadium 3 - rasch zunehmende Verbreitung von Tabakerzeugnissen mit modifiziertem Risiko in Ländern, die bei der Tabakkontrolle schon relativ weit sind, in denen Absatz und Konsum von Zigaretten stetig sinken und in denen Strategien zur Schadensminderung auf hohe Akzeptanz und rasch zunehmende Verbreitung stoßen. In Stadium-3-Ländern wirft die plötzlich aufkommende E-Zigarettenexposition der Bevölkerung aus Sicht des Gesundheitswesens ein moralisches Dilemma auf. Auf der einen Seite könnte die E-Zigarette eine Zeitenwende in der Raucherentwöhnung bzw. als Teil einer Schadensminderungsstrategie einläuten. Auf der anderen Seite ist sie ein unzureichend geprüftes Produkt, das die Nikotinsucht verstärken oder wieder normalisieren könnte und potenziell die Wirksamkeit der evidenzbasierten Maßnahmen zur Tabakbekämpfung untergräbt, mit deren Hilfe in den letzten Jahrzehnten ein Rückgang der Raucherquoten erreicht wurde. Eine der Hauptfragen aus Sicht des Gesundheitswesens lautet daher, ob E-Zigaretten einen potenziellen Einstieg in die Nikotinsucht und den nachfolgenden Tabakkonsum darstellen. Abschließend gibt das Kapitel einen Überblick über die Regulierungsmaßnahmen für EZigaretten, die im Rahmen einer umfassenden Strategie zur Tabakkontrolle erforderlich sind.

(c) 2017 S. Karger GmbH, Freiburg
Alles fließt, nichts bleibt

Heraklit

(540-480 v. Chr.)

Veränderung ist ein fester Bestandteil aller biologischen, internationalen und gesellschaftlichen Systeme. Ebenso entwickeln sich menschliche Erfindungen und Produkte im Zuge des technischen Fortschritts und sozialen Wandels weiter. Das erste Telefon
z.B. erfand Alexander Graham Bell in den 1870er Jahren, um Sprache übertragen zu können - ein simples Gerät, dessen Weiterentwicklung über das Festnetztelefon in jedem Haushalt und die ersten Mobiltelefone der 1980er Jahre zu den schlanken Alleskönner-Smartphones von heute führte, mit denen man nicht nur telefonieren, sondern auch Daten, Videos und Textnachrichten übertragen und Tausende Software-Applikationen nutzen kann. Ähnlich haben sich auch Tabakwaren und -konsum im Laufe der

\section{KARGER}

Fax +497614520714

information@karger.com

www.karger.com

\section{(c) 2017 S. Karger GmbH, Freiburg}

Accessible online at:

www.karger.com/kkd
Constantine I. Vardavas

Center for Global Tobacco Control, Department of Social and Behavioral Sciences

Harvard School of Public Health

401 Park Drive, 4th West, Boston, 02215 MA, USA

vardavas@ tobcontrol.eu 
Jahrhunderte stark verändert - vom Pfeiferauchen bei Heil- und Festritualen im vorkolumbischen Amerika über industriell produzierte klassische Zigaretten bis hin zu verschiedensten neuen (und potenziell noch stärker süchtig machenden) Produkten, von denen jedes als besser oder zumindest potenziell weniger schädlich angepriesen wurde als die Vorgänger. Elektrische Zigaretten (E-Zigaretten) sind neben anderen nikotinhaltigen Produkten, die weitgehend seit den späten 2000er Jahren im Handel sind, die neueste Stufe in dieser Entwicklung und werden in der internationalen Fachwelt des Gesundheitswesens vielfach kontrovers diskutiert [1]. Dieser Beitrag ist der Versuch einer ganzheitlichen Betrachtung verschiedener von Wissenschaft und Politik aufgeworfener Fragen zur Gestaltung, Herstellung, Vermarktung und Nutzung von E-Zigaretten und den daraus folgenden Auswirkungen auf tabakbezogene Outcomes für den Einzelnen und die Gesellschaft.

\section{Entwicklung der Tabakepidemie und Aufstieg der elektrischen Zigarette}

Die klassischen Stadien des Tabakkonsums in einer Gesellschaft mitsamt der damit einhergehenden Mortalität wurden 1994 von Lopez et al. [2] beschrieben; sie charakterisieren vier Stadien anhand der Assoziation zwischen Konsumprävalenz bei Erwachsenen beiderlei Geschlechts und dem Zeitrahmen, in dem die tabakbedingte Mortalität bei beiden Geschlechtern ihren Höhepunkt erreicht.

Wir schlagen ein alternatives neues Modell der Tabakepidemie mit drei Stadien vor, deren charakteristische Merkmale die Art der vorwiegend verwendeten Tabakprodukte und der Stand in Bezug zur globalen Tabakepidemie sind. Dieses Modell basiert auf der Hypothese, dass das Paradigma der Infektionskrankheiten auch auf den Tabakkonsum übertragbar ist (Abb. 1) [3]. Genau wie die Mutation eines Infektionserregers (z.B. eines Virus) dessen Infektiosität, Pathogenität und Übertragbarkeit für die Wirtsorganismen beeinflussen kann, so beeinflusst dieser Hypothese zufolge auch die Weiterentwicklung («Mutation») von Tabakerzeugnissen («Infektionserregern») durch die Tabakindustrie (den «Überträger») das Einstiegs- und Suchtpotenzial («Infektiosität») von Tabakerzeugnissen bei Jugendlichen und jungen Erwachsenen (den «Wirten»). Und ebenso wie verschiedene Viren je nach Prädisposition verschiedene Zellen und Organe angreifen, können unterschiedliche Arten des Tabakkonsums sich auf verschiedene Personen («Zellen») und Gesellschaften («Organe») unterschiedlich auswirken.

\section{Ein neues Drei-Stadien-Modell der Tabakepidemie}

Stadium 1: Überwiegende Verwendung konventioneller Tabakerzeugnisse

Länder im Stadium 1 der globalen Tabakepidemie befinden sich in der frühen Phase des Tabakkonsums. In diesen Ländern ist die Nutzung von Tabakerzeugnissen in der Bevölkerung noch im Anstieg - entweder, weil internationale Tabakkonzerne erst kürzlich den lokalen Markt eingedrungen sind, oder vor einem kulturellen Hintergrund traditioneller Formen des Tabakkonsums,
Abb. 1. Epidemiemodell der Nikotinsucht und Tabakkontrolle (nach Orleans und Slade [68] (Übersetzung), mit freundlicher Genehmigung der Oxford University Press, USA).

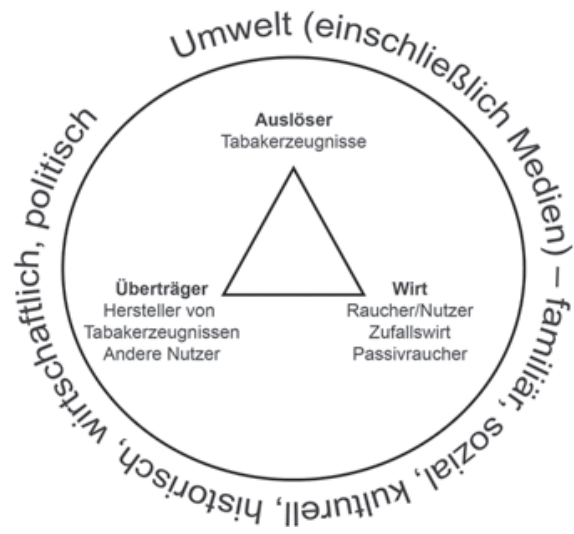

z.B. Kretek, Wasserpfeife, Kau- oder Schnupftabak. Hierunter fallen derzeit verschiedene Länder in Südostasien, China, Länder des Nahen Ostens sowie die afrikanische Region.

Stadium 2: Nebeneinander von konventionellen Tabakerzeugnissen und solchen mit modifiziertem Risiko

In Ländern im Stadium 2 der globalen Tabakepidemie gibt es bereits zunehmend Initiativen zur Tabakkontrolle, doch die epidemiebedingte Mortalität bei Männern und Frauen ist noch an ihrem Höhepunkt. In dem Maße, wie in diesen Ländern das Bewusstsein für die schädlichen Auswirkungen des Tabakkonsums zunimmt und die Bemühungen im Kampf gegen den Tabak verstärkt werden, steigt potenziell auch das Interesse der Bevölkerung an Strategien zur Schadensminderung und deren praktischer Umsetzung. Zu den Ländern in diesem Stadium zählen die Länder Süd- und Osteuropas sowie einige asiatische und südamerikanische Länder.

Stadium 3: Rasch zunehmende Verbreitung von Tabakerzeugnissen mit modifiziertem Risiko

Die Länder im Stadium 3 der globalen Tabakepidemie sind bei der Tabakkontrolle schon relativ weit gekommen. Dieses Stadium ist durch sinkenden Absatz und Konsum von Zigaretten gekennzeichnet, möglicherweise dank eines geschärften Bewusstseins der Bevölkerung für die schädlichen Wirkungen von Tabak sowie umfassende Tabakkontrollprogramme. In diesen Ländern stoßen Strategien zur Schadensminderung auf hohe Akzeptanz und rasch zunehmende Verbreitung. Beispiele für die Länder im Stadium 3 sind die USA, Kanada, Australien und verschiedene Länder in Nordwesteuropa.

Die nun plötzlich aufkommende E-Zigarettenexposition der Bevölkerung in Stadium-3-Ländern wirft aus Sicht des Gesundheitswesens ein moralisches Dilemma auf. Auf der einen Seite könnte die E-Zigarette eine Zeitenwende in der Raucherentwöhnung bzw. als Teil einer Schadensminderungsstrategie einläuten; auf der anderen Seite ist sie ein unzureichend geprüftes Produkt, das die Nikotinsucht verstärken oder wieder normalisieren könnte und potenziell die Wirksamkeit der evidenzbasierten Maßnahmen zur Tabakbekämpfung untergräbt, mit deren Hilfe in den letzten Jahrzehnten ein Rückgang der Raucherquoten erreicht wurde [4-6]. 


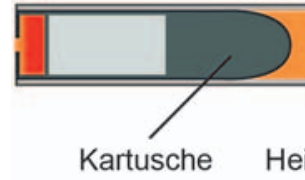

Kartusche

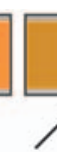

Heizwendel

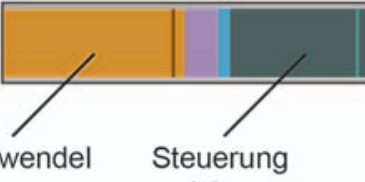

und Sensor

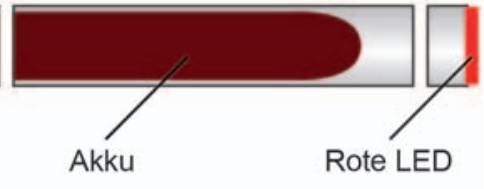

\section{Das Prinzip der E-Zigarette}

Was ist überhaupt eine E-Zigarette? E-Zigaretten sind eine Klasse meist akkubetriebener Geräte (Abb. 2), in denen eine chemische Mischung (das Liquid) zu einem Aerosol verdampft wird. Das Liquid enthält typischerweise Nikotin, Aromastoffe und andere funktionelle Bestandteile in einer Trägerflüssigkeit - meist Propylenglykol oder Glycerin. Das Liquid wandert durch einen Verdampfer (das Heizelement) und wird dort in Nebel umgewandelt, den der Nutzer dann inhaliert. Während dies das grundlegende Konzept ist, das alle E-Zigaretten gemeinsam haben, gibt es inzwischen ein breites Spektrum unterschiedlicher Produkttypen und Marken in verschiedenen Formen, Größen und Geschmacksrichtungen - daher die obige Definition von «E-Zigarette» als Sammelbegriff für eine ganze Klasse von Produkten. Die drei gängigsten Arten von E-Zigaretten auf dem EU- und US-Markt sind Einweg-E-Zigaretten, nachfüllbare und wiederaufladbare EZigaretten (einschließlich solcher mit «Tank») sowie sogenannte Mods, die sich dadurch auszeichnen, dass sie vom Nutzer modifiziert werden können. Einweg-E-Zigaretten haben eine nicht wiederaufladbare Batterie und werden im Ganzen entsorgt, sobald die Flüssigkeit aufgebraucht ist; wiederaufladbare E-Zigaretten haben einen wiederaufladbaren Akku und entweder auswechselbare Liquid-Kartuschen oder nachfüllbare Tanks; und Mods sind wiederaufladbare E-Zigaretten, bei denen der Nutzer nach seinem Geschmack den Aufbau anpassen und/oder die Funktion regulieren kann. Die letzteren gelten als Geräte der neueren Generation.

\section{Inhaltsstoffe der E-Zigarette}

\section{Nikotin}

Die Nikotinabgabe ist einer der wichtigsten Aspekte bei der Gestaltung von E-Zigaretten. Das Ausmaß der Nikotinabgabe in EZigaretten bildet die biologische Grundlage für die Nikotinsucht des Nutzers einerseits und für den potenziellen Einsatz der E-Zigarette in der Pharmakotherapie der Nikotinabhängigkeit andererseits. Der Nikotingehalt des Liquids ist nur einer von mehreren Faktoren, die die Nikotinabgabe beeinflussen. Weitere Faktoren sind die Geschwindigkeit und das Areal der Resorption in den Atemwegen - was wiederum unmittelbar von der Topographie und Nutzungsart des Produkts abhängig ist. Die höchsten Nikotinwerte, die mit E-Zigaretten früher Generationen erreicht wurden, lagen Untersuchungen zufolge im unteren Bereich der Werte von Produkten für die orale Nikotinersatztherapie (NET) und deutlich unterhalb der Werte von herkömmlichen Zigaretten [7]. Möglicherweise erfolgt die Nikotinaufnahme beim Dampfen von
E-Zigaretten derzeit zu einem höheren Anteil durch Resorption über die Schleimhäute - ein langsamer ablaufender Prozess, wie beim Konsum von rauchfreien Tabakprodukten. Beim klassischen Zigarettenrauchen hingegen kommt es zu einer schnelleren arteriellen Nikotinresorption (dem «Spike»), weil der Zigarettenrauch nach dem Inhalieren tiefer in die Lungen eindringt $[8,9]$. Auf kurze Sicht wird wahrscheinlich die E-Zigarette von der Industrie auf eine schnellere Nikotinresorption ausgerichtet werden, um möglichst die Nikotinaufnahme von herkömmlichen Zigaretten zu imitieren.

Nutzungsmerkmale und Topographie können ebenfalls die Nikotinaufnahme beeinflussen, da sich die Topographie beim Dampfen einer E-Zigarette erheblich von der beim herkömmlichen Zigarettenrauchen unterscheidet: Die Züge haben ein größeres Volumen, dauern länger, und es wird für eine bestimmte Nikotindosis eine höhere Anzahl an Zügen benötigt [10]. Auch die Erfahrenheit des Nutzers spielt eine Rolle; erfahrene E-Zigarettenkonsumenten kommen eher auf höhere Nikotindosen als unerfahrene Nutzer [11].

\section{Trägerflüssigkeit}

Die gebräuchlichsten Trägerflüssigkeiten in Liquids für E-Zigaretten sind Propylenglykol und pflanzliches Glycerin; entweder eins allein oder beide als Gemisch (in unterschiedlichen Anteilen). Die Inhalation der Trägerflüssigkeit ist nicht uneingeschränkt sicher, insbesondere für unerfahrene Nutzer von E-Zigaretten, die bisweilen ein größeres Dampfvolumen inhalieren, um den gewünschten Nikotin-«Kick» zu erhalten [12]. Insbesondere wurde gezeigt, dass die Trägerflüssigkeit Ausgangsbasis für die Bildung sekundärer toxischer Verbindungen sein kann. Während der Verdampfung kann es durch thermische Zersetzung der Trägerflüssigkeit im Zerstäuber zur Bildung von niedermolekularen Carbonylverbindungen kommen, die bekanntermaßen toxische Eigenschaften besitzen, wie z.B. Formaldehyd, Acetaldehyd und Acrolein $[12,13]$. Zur Bildung dieser potenziell schädlichen Nebenprodukte kommt es nachweislich insbesondere bei E-Zigaretten mit höherer Spannung (und dadurch höherer Temperatur) sowie bei Verwendung von Propylenglykol als Trägerflüssigkeit [14].

\section{Geschmackszusätze}

Durch Untersuchungen der Tabakindustrie ist umfassend gesichert, dass die sensorischen Merkmale, wie der Geschmack, an der Beurteilung beteiligt sind, wie attraktiv und angenehm das Produkt wahrgenommen wird. Sie sind damit maßgebliche Einflussfaktoren für das Rauchverhalten, über die unmittelbare Wirkung des Nikotins hinaus [15]. Der Geschmack kann sekundär 
suchtverstärkend wirken, insbesondere bei Jugendlichen und jungen Erwachsenen. Eine Fülle charakterisierender Aromen stehen für E-Zigaretten zur Wahl, und sie tragen potenziell dazu bei, die Nutzung zu erleichtern, die Entwöhnung zu unterdrücken und einen antizipatorischen Belohnungseffekt zu erzeugen [16].

\section{Verunreinigungen}

In Untersuchungen wurden Abweichungen zwischen den angegebenen Mengen der Inhaltsstoffe und dem tatsächlich im Liquid bzw. Dampf gemessenen Gehalt festgestellt [17]. Es gibt auch Hinweise darauf, dass bei E-Zigaretten früherer Generationen die Kartuschen, Verdampfer und Dämpfe Spuren verschiedener Verunreinigungen enthalten können. Vorliegende Studien belegen, dass der Dampf von E-Zigaretten variierende Mengen an Carbonylverbindungen (z.B. Formaldehyd, Acetaldehyd, Acrolein), flüchtige organische Verbindungen (z.B. Toluol) und tabakspezifische Nitrosamine enthalten kann, mit erheblichen Unterschieden je nach Marke [14, 17]. Der Gehalt ist zwar wesentlich niedriger als in den Emissionen konventioneller Zigaretten, aber immer noch bedenklich für die Gesundheit des Nutzers. Abhilfe gegen die Abweichungen bei den Wertangaben sowie die mögliche Schädigung durch Verunreinigungen könnten die Einführung strenger Qualitätssicherungsverfahren und andere Umstellungen in der Produktion sowie die Entwicklung von standardisierten Messprotokollen schaffen.

\section{Auswirkungen von E-Zigaretten auf den Einzelnen}

Da Verbrennung und verschiedene Emissionen entfallen, sind E-Zigaretten höchstwahrscheinlich erheblich weniger schädlich für die Atemwege als konventionelle Zigaretten [12, 18]. Die kurzund langfristigen Auswirkungen der Inhalation von Trägerflüssigkeit und Nikotin aus E-Zigaretten müssen jedoch noch weitergehend untersucht werden $[19,20]$. Die bisherigen Forschungsergebnisse deuten darauf hin, dass im Gegensatz zum konventionellen Zigarettenrauchen die akute Nutzung von E-Zigaretten sich nicht auf Lungenfunktionswerte wie Einsekundenkapazität oder forcierte Vitalkapazität auswirkt [21]. In Laboruntersuchungen war die akute Nutzung von E-Zigaretten allerdings mit einer kurzfristigen Erhöhung des Gefäßwiderstand der kleinen Lungengefäße assoziiert (gemessen mittels Impulsoszillometrie, einem Verfahren, das von der Mitwirkung des Untersuchten unbeeinflusst bleibt und empfindlicher auf geringfügige Veränderungen reagiert als die klassische Spirometrie), außerdem mit einer Abnahme der Stickstoffmonoxidkonzentration in der Ausatemluft und einer Verringerung der SpO2-Konzentration [22-24]. Hierbei ist allerdings anzumerken, dass zwar bei allen diesen $\mathrm{Pa}-$ rametern statistisch signifikante Unterschiede gemessen wurden, jedoch die klinische Bedeutung im Hinblick auf die Initiierung oder Verschlechterung von Atemwegserkrankungen weitgehend ungeklärt ist [22]. Wie E-Zigaretten sich auf die Lungengesundheit von Bevölkerungsgruppen auswirken, die besonders gefährdet für das Tabakrauchen sind, ist Gegenstand lebhafter Diskussionen. Einerseits lässt sich anführen, dass Zigarettenraucher, die vollständig auf E-Zigaretten umsteigen, ihr Risiko für tabakbedingte Krankheiten verringern. Andererseits sind diese Personen im Vergleich zur Allgemeinbevölkerung möglicherweise anfälliger für potenzielle Nebenwirkungen von E-Zigaretten. Im Tiermodell wurde gezeigt, dass die Inhalation von E-ZigarettenLiquids Allergiesymptome verstärken kann [25]. Erste Studienergebnisse zum pulmonalen Gefäßwiderstand bei menschlichen Probanden mit chronisch-obstruktiver Lungenerkrankung oder Asthma belegen allerdings ein begrenztes Maß an Veränderungen $[23,24]$.

Die Auswirkungen von E-Zigaretten auf die Herzfunktion sind noch relativ unerforscht. Theoretisch könnte die Nutzung von E-Zigaretten auf verschiedenen Wegen die Herzfunktion beeinträchtigen: durch den Nikotingehalt sowie den Gehalt an Feinstaubpartikeln (meist unter $100 \mathrm{~nm}$ ) und Nanopartikeln (mehr als die Hälfte der bei der E-Zigarettennutzung entstehenden Partikel) $[8,11,17]$. Bisher sind mögliche Assoziationen jedoch kaum erforscht worden. In Tiermodellen wurde gezeigt, dass Nikotin eine Vielzahl physiologischer Wirkungen über Nikotinacetylcholinrezeptoren auslöst und das Wachstum von atherosklerotischen Läsionen fördert. Allerdings haben Studien beim Menschen zu NET oder oralen Tabakprodukten keinen solchen $\mathrm{Zu}$ sammenhang ergeben. Es ist jedoch möglich, dass der andere Expositionsweg (orale Einnahme/transdermale Applikation vs. Inhalation) und das Vorliegen von Nanopartikeln und Feinstaubpartikeln die beobachteten Zusammenhänge beeinflussen könnten. Bisher wurden erst in einer einzigen Studie bei gesunden Probanden im Alter von 20 bis 45 Jahren kardiovaskuläre Indizes im Zusammenhang mit der Nutzung von E-Zigaretten betrachtet. Hier wurden bei keinem echokardiographischen $\mathrm{Pa}-$ rameter signifikante Veränderungen festgestellt, mit Ausnahme einer leichten Erhöhung bei einem Parameter [18]. Andere klinische Studien haben ergeben, dass E-Zigaretten sich nicht auf zirkulierende Entzündungsmarker, Herzfrequenz oder Blutwerte auswirken [26].

Studien zur Zytotoxizität von E-Zigaretten-Liquid lieferten widersprüchliche Ergebnisse. Bahl et al. [27] untersuchten 40 Proben der Verbrauchsflüssigkeit für E-Zigaretten an menschlichen Embryo- und murinen neuralen Stammzellen sowie adulten menschlichen Lungenfibroblasten; hier erwiesen sich die ersteren beiden als empfindlicher gegenüber den Liquids als die adulten Zellen. In dieser Studie korrelierte die Zytotoxizität signifikant mit der Anzahl und Konzentration von chemischen Geschmacksstoffen, war aber nicht signifikant mit dem Typ der Trägerflüssigkeit assoziiert, und im Hinblick auf Nikotin waren die Ergebnisse inkonsistent. In ähnlicher Weise stellten Romagna et al. [28] bei den meisten untersuchten Proben keine Zytotoxizität fest, mit Ausnahme von einer Probe mit erhöhter Konzentration an Kaffeearoma. Eine andere Studie ergab Hinweise darauf, dass einige E-Zigarettenproben für kultivierte Kardiomyoblasten zytotoxische Eigenschaften haben; diese sind mit dem Produktionsprozess und den für die Aromen verwendeten Materialien assoziiert [29]. Diese Ergebnisse sind insofern von besonderem Interesse, als es eine Fülle von Aromen für E-Zigaretten gibt - schätzungsweise 
7700 verschiedene Geschmacksrichtungen von 466 Marken [30]. In einer weiteren Studie wurde darüber hinaus die potenzielle $\mathrm{Zy}$ totoxizität anderer in Spuren vorliegender Verunreinigungen untersucht, z.B. Zinn [31].

\section{Auswirkungen von E-Zigaretten auf die Gesellschaft}

\section{Auswirkung auf Raucher: Schadensminderung}

Nachdem es in diesem Beitrag bisher vorwiegend um die Risiken der Nutzung von E-Zigaretten ging, soll im Sinne der Ausgewogenheit auch die mögliche Rolle der E-Zigarette im Rahmen einer Strategie zur Schadensminderung nicht unerwähnt bleiben. Das Prinzip der Schadensminderung ist in Bezug auf die Tabakkontrolle der Ansatz, über den regulatorische Maßnahmen der öffentlichen Hand auf die Reduktion der schädlichen Folgen des Tabakkonsums abzielen - in diesem Fall durch die steigende Verbreitung von vergleichsweise weniger schädlichen nikotinbasierten Produkten. Das Konzept der Schadensminderung ist seit jeher umstritten und sorgt in der Fachwelt für erhebliche Diskussionen; so auch jetzt im Falle der E-Zigarette. E-Zigaretten sind so konzipiert, dass sie ohne die Verbrennung von Tabak auskommen; dadurch ist es weniger wahrscheinlich als bei brennbaren Tabakprodukten, dass sie eine unmittelbare Gefahr für den Nutzer darstellen. Wenn ein Raucher von Zigaretten vollständig auf E-Zigarette umsteigt, könnte er dadurch möglicherweise sein Risiko für tabakbedingte Morbidität und Mortalität verringern; man könnte somit argumentieren, dass die E-Zigarette einen individuellen Nutzen durch Schadensminderung bringt. Darüber hinaus belegen Laborexperimente eine signifikant geringere Belastung durch toxische Chemikalien in E-Zigarettendampf im Vergleich zu herkömmlichem Zigarettenrauch [17, 32, 33]. Um einen populationsweiten Effekt zu erzielen, müssten E-Zigaretten jedoch reguliert werden, um sicherzustellen, dass ihre Nutzung zu höheren Raten vollständiger Entwöhnung und zu sinkender Intensität des Tabakkonsums führt und nicht den dualen Konsum fördert. Da rauchbedingte Herz-Kreislauf- und Krebsrisiken stark von der Dauer des Tabakkonsums und nicht nur von der Intensität (Zahl der Zigaretten pro Tag) abhängen, wird derzeit diskutiert, ob duale Konsumenten gesundheitlich signifikant profitieren können, wenn sie weiterhin einige wenige Zigaretten pro Tag rauchen $[34,35]$.

E-Zigaretten als Mittel, um nicht mehr bzw. weniger zu rauchen Mehrere Studien belegen, dass die Nutzung von E-Zigaretten dazu führen kann, dass weniger konventionelle Zigaretten pro Tag geraucht werden $[36,37]$, und bisher hat eine randomisierte kontrollierte Studie Hinweise darauf ergeben, dass E-Zigaretten mindestens so hilfreich bei der Raucherentwöhnung sind wie eine NET [38]. In einer kürzlich in Großbritannien durchgeführten Querschnittstudie war bei Rauchern, die E-Zigaretten nutzten, die Wahrscheinlichkeit höher, dass sie langfristig abstinent blieben, als bei Rauchern, die einen Entwöhnungsversuch ohne angeleitete NET oder ohne professionelle Unterstützung unternahmen [39]. In unserer Sekundäranalyse der Eurobarometer-Daten von 2012 (der unseres Wissens größten populationsbasierten Stu- die) gelangten wir zu dem Ergebnis, dass - nach Bereinigung um soziodemographische Merkmale, geographische Region und Zigarettenpräferenzen - aktuelle Raucher, die im vorherigen Jahr einen Entwöhnungsversuch unternommen hatten, doppelt so häufig E-Zigaretten ausprobiert hatten wie Raucher, die nicht versucht hatten aufzuhören [40]. Im Gegensatz dazu ergab eine aktuelle Metaanalyse von fünf anderen populationsbasierten Studien, dass bei aktuellen Nutzern von E-Zigaretten die Wahrscheinlichkeit, dass sie aufhörten, 39\% geringer war als bei Rauchern, die keine E-Zigaretten nutzten [41]. Dies deckt sich mit dem Bericht des US Institute of Medicine, dem zufolge die Verfügbarkeit anderer Tabakerzeugnisse sich im Saldo negativ auf die öffentliche Gesundheit auswirken könnte, wenn Raucher nicht oder später aufhören, Ex-Raucher rückfällig werden und Nichtraucher möglicherweise eher ermutigt werden, anzufangen [42].

Zusammengenommen deuten diese Befunde möglicherweise daraufhin, dass E-Zigaretten potenziell ein zweischneidiges Schwert sind - einerseits könnten sie von Rauchern, die bereits hinreichend zum Aufhören motiviert sind, als Hilfe zur Entwöhnung genutzt werden (ähnlich wie NET, aber mit den Verhaltensmustern/Situationen wie beim Rauchen) [43], andererseits könnten weniger entwöhnungsmotivierte Raucher E-Zigaretten parallel zum konventionellen Zigarettenrauchen nutzen (dualer Konsum) und dadurch mit geringerer Wahrscheinlichkeit ganz aufhören.

\section{Auswirkungen auf Ex-Raucher}

Über die möglichen Effekte der Verfügbarkeit von E-Zigaretten auf die Rückfallwahrscheinlichkeit des Zigarettenrauchens kann man derzeit nur Vermutungen anstellen. Entsprechende Hypothesen sind erst in wenigen Langzeitstudien geprüft worden. Einige haben Hinweise darauf ergeben, dass ein gewisser Anteil der Ex-Raucher E-Zigaretten ausprobieren könnte; allerdings waren die Stichprobengrößen dieser Untergruppen zu klein, um belastbare Schlussfolgerungen zuzulassen [44]. In Querschnittstudien (bei denen keine Ursache-Wirkung-Beziehungen hergestellt werden können) wurde festgestellt, dass ein Teil der Ex-Raucher EZigaretten ausprobiert hat (nämlich 2,7\% in Großbritannien bzw. $4 \%$ in der gesamten EU), doch mit diesem Studiendesign lässt sich nicht erkennen, ob die jeweilige Person E-Zigaretten ausprobierte, während sie noch rauchte, und danach aufhörte (mit oder ohne Nutzung von E-Zigaretten) oder ob sie als Ex-Raucher zur E-Zigarette griff und dies den Wiedereinstieg in die Nikotinsucht bedeutete $[40,45]$.

\section{Auswirkungen auf Nichtraucher}

Eine der Hauptfragen aus Sicht des Gesundheitswesens ist die, ob E-Zigaretten einen potenziellen Einstieg in die Nikotinsucht und den nachfolgenden Tabakkonsum darstellen $[41,46]$. Bisher wurden nur in Querschnittstudien die Zusammenhänge zwischen EZigaretten und Anfälligkeit für sowie Ausprobieren oder Angewöhnen von konventionellem Zigarettenrauchen unter Nichtrauchern untersucht. Der Anteil der Erwachsenen, die schon einmal eine E-Zigarette gedampft hatten, wurde in Großbritannien auf $0,5 \%$ geschätzt, in der EU auf $1,2 \%$ und in den USA auf 0,8-3,8\%; 
außerdem bei Teenagern in den USA auf 0,9-4,4\% und in Korea auf $1,4 \%$ [34, 45, 47-52]. Diese Zahlen zeigen zwar eine relativ niedrige (wenngleich steigende) Verbreitung von E-Zigaretten unter Personen, die zuvor nie geraucht haben, doch das NettoPopulationsrisiko im Verhältnis zu diesem Anstieg ist noch ungeklärt. Somit ist auch die Gesamtauswirkung der E-Zigarette auf die öffentliche Gesundheit unbekannt, und deshalb ist weiterhin das Vorsichtsprinzip geboten [53-55].

Eine weitere Gefahr im Zusammenhang mit der Einführung von E-Zigaretten sind unbeabsichtigte Vergiftungen bei Kindern. Eine aktuelle Studie in den USA zeigt für E-Zigaretten und konventionelle Zigaretten zusammengenommen eine Zunahme der Anrufe bei Giftinformationszentren von 0,3\% im September 2010 auf 41,7\% im Februar 2014 [56]. Die häufigsten Formen der Exposition gegenüber E-Zigaretten in den USA waren die Einnahme von Liquid (69\%), dessen Inhalation (17\%) oder die Aufnahme über die Augen (9\%) oder die Haut (6\%); dies konnten Unfälle bei Kindern sein oder potenziell auch bei E-Zigarettennutzern während des Nachfüllvorgangs [56, 57]. Der US Surgeon General's Report von 2014 weist darauf hin, dass ausreichende Evidenz vorliegt, um zu dem Schluss zu gelangen, dass Nikotin in ausreichend hohen Dosen akute Toxizität hervorruft; dies unterstreicht die Notwendigkeit, solchen versehentlichen Expositionen vorzubeugen, indem man den Aufbau und den Nachfüllvorgang von E-Zigaretten verändert [58].

\section{Direkter und indirekter Passivdampf}

Die Schadstoffkonzentrationen im Dampf von E-Zigaretten sind zwar signifikant niedriger als in demselben Volumen von Zigarettenrauch, doch die Werte deuten darauf hin, dass weder E-Zigaretten vollständig emissionsfrei sind noch ihr Dampf vollkommen ungefährlich [12, 14, 18, 32, 59]. Kürzlich wurde in einer ersten Studie unter Alltagsbedingungen die Nutzung von E-Zigaretten im häuslichen Umfeld mit erhöhten Mengen an Nikotin in der Luft in den Wohnräumen in Beziehung gesetzt: Der Nikotingehalt der Luft war zwar bei den E-Zigarettennutzern nicht so hoch wie in den Wohnräumen von Zigarettenrauchern, aber dennoch signifikant höher als bei Nichtrauchern [60]. Darüber hinaus war die Nutzung von E-Zigaretten auch mit einer erhöhten Wahrscheinlichkeit für indirekte passive Nikotinexposition über Oberflächen assoziiert, wobei das Ausmaß der Exposition je nach Art der Oberfläche und Marke der E-Zigarette variierte [61]. Potenziell gesundheitsschädliche Nebenprodukte der E-Zigarette wie Acrolein, polyzyklische aromatische Kohlenwasserstoffe, Carbonylverbindungen, Schwermetalle und Feinstaubpartikel könnten möglicherweise durch Veränderungen im Aufbau und in der Technik der E-Zigarette reduziert werden.

\section{E-Zigaretten aus regulatorischer Sicht}

Es besteht dringender Bedarf an wissenschaftlicher Forschung zu regulatorischen Fragen, um eine ausgewogene Regulierung von E-Zigaretten zu finden. $\mathrm{Zu}$ den Aspekten, zu denen eine breitere Evidenzbasis benötigt wird, bevor rechtliche Regelungen festgelegt werden können, zählen die kurz- und langfristigen Folgen der
Tab. 1. Empfehlungen des Forum of International Respiratory Societies zu E-Zigaretten [62]
1) Verbot von Werbung, Promotion und Sponsoring
2) Auslageverbot im Einzelhandel
3) Verbot des Verkaufs an Minderjährige
4) Regulierung des Onlinevertriebs
5) Besteuerung zu ähnlichen Sätzen wie konventionelle Zigaretten
6) Verbot des Verkaufs von und Anreicherung mit Aromastoffen, die attraktiv für Kinder und Jugendliche sind
7) Verpflichtung der Deklaration aller Inhaltsstoffe und des Nikotingehalts auf der Verpackung
8) Verpflichtung zur Anbringung adäquater Warnhinweise (wie für Tabakerzeugnisse)
9) Verbot der Nutzung in öffentlichen Räumen, am Arbeitsplatz und in öffentlichen Verkehrsmitteln

Nutzung von E-Zigaretten, ihr Nikotinabgabepotenzial sowie eine spezifische Beurteilung ihrer möglichen Auswirkungen für den Einzelnen und die Gesellschaft. Das Forum of International Respiratory Societies empfiehlt, elektrische Nikotinabgabesysteme, sofern sie nicht als Arzneimittel/Medizinprodukte besteuert werden, als Tabakprodukte zu regulieren (Tab. 1) [62]. Ähnliche Empfehlungen finden sich in umfassenden Positionspapern der American Heart Association [63] und der American Association for Cancer Research/American Society of Clinical Oncology [64]. E-Zigaretten sollten reguliert werden, um zu verhindern, dass sie den dualen Konsum und den Einstieg von Jugendlichen fördern und die Nichtraucherschutzgesetze und andere politische Maßnahmen zur Tabakkontrolle unterminieren. Angesichts dessen, dass diese sich schnell entwickelnde Produktklasse sehr wahrscheinlich erhebliche Einwirkung auf Tabakkonsum und -regulierung haben wird, ist es zwingend notwendig, regulatorische Maßnahmen zu identifizieren, die in verschiedenen Ländern bereits in unterschiedlichem Maße implementiert wurden, um sie in der $\mathrm{Zu}$ kunft weiter zu stärken. Von diesen Maßnahmen könnten auch Länder profitieren, die eine Regulierung für E-Zigaretten im Rahmen einer umfassenden Tabakkontrollpolitik einführen wollen [62-65]. Diese Maßnahmen lassen sich wie folgt zusammenfassen:

\section{1) Verbot der Nutzung in öffentlichen Räumen}

Jegliche Nutzung von E-Zigaretten ist in allen öffentlichen Bereichen zu untersagen, in denen auch der Konsum konventioneller Tabakerzeugnisse untersagt ist, um nicht den Nutzen von Nichtraucherbereichen zu untergraben, um die Raucherentwöhnung zu fördern und zu erleichtern. Die Nutzung von E-Zigaretten in Bereichen zu gestatten, in denen das Rauchen nicht gestattet ist, würde gegen Artikel 8 und 12 der WHORahmenkonvention zur Tabakkontrolle (FCTC) verstoßen, da E-Zigaretten zur unfreiwilligen Passiv-Exposition von Nichtrauchern und auch zur erneuten Akzeptanzzunahmen des Tabakkonsums führen können.

(2) Verbot von Werbung und Verkaufsförderung Dies gilt auch für die Verpackung, Kennzeichnung, Vermarktung und Bewerbung von E-Zigaretten. Eine kürzlich veröf- 
fentlichte Entscheidung der WHO, die während der sechsten Konferenz der Vertragsparteien gefasst wurde, fordert die Parteien auf, Verbote bzw. Beschränkungen für Werbung, Promotion und Sponsoring für elektrische Nikotinabgabesysteme (ENDS) in Betracht zu ziehen und zu verhindern, dass Nichtraucher und Jugendliche mit der Nutzung von ENDS/ nikotinfreien Inhalationssystemen (ENNDS) anfangen, mit besonderem Augenmerk auf überdurchschnittlich gefährdeten Gruppen. Dies ist ein wichtiger Aspekt der neuen Produktregulierung - vor allem, wenn die Vermarktung als Produkt nur für Raucher erfolgt. Derzeit werden gesundheitliche Aspekte und Raucherentwöhnung häufig als Verkaufsargument für E-Zigaretten angeführt, obwohl keine stichhaltigen wissenschaftlichen Beweise vorliegen; außerdem werden häufig Aussagen von Ärzten auf Websites verwendet und Prominente als Botschafter gewonnen [66] - mit unbekannten Folgen für die Verbraucher.

3) Angabe und Regulierung der Bestandteile mit standardisierten Protokollen für die Messung und Angabe

Sowohl die EU als auch die USA entwickeln derzeit Richtlinien für die Angabe der Inhaltsstoffe und Emissionen von E-Zigaretten. Sollten die Bestandteile durch ein einheitliches Angabeformat und durch Verbesserungen bei Produktion und Handhabung reguliert werden, wäre es möglich, gesundheitsschädliche Nebenprodukte zu reduzieren, einschließlich der Bestandteile von E-Zigarettenemissionen. Dieser Prozess wird - zumindest in der EU - dadurch erleichtert, dass die Angabe der Inhaltsstoffe und Emissionen von E-Zigaretten gegenüber den Behörden der Mitgliedstaaten und der Europäischen Kommission in Artikel 7 und 20 der EU-Richtlinie über Tabakerzeugnisse (2014/40/EU) vorgeschrieben ist [67].

4) Schutz von Kindern und besonders gefährdeten Bevölkerungsgruppen vor unfreiwilliger Exposition

Die Verpackungen von E-Zigaretten und die dazugehörigen Fläschchen sollten kinder- und manipulationssicher sein, um unbeabsichtigte Exposition oder Aufnahme durch Kinder zu vermeiden. Dieser Aspekt lässt sich leicht regulieren und durchsetzen, indem man ihn in die vorgeschriebenen Voraussetzungen für die Vermarktung aufnimmt. Die EU-Richtlinie über Tabakerzeugnisse ebnet mit Artikel 20 den Weg für eine solche Vorgehensweise - eine Neuerung, der wir positiv entgegensehen [67].

5) Schutz der Gesetzgebung vor Branchenlobbyismus

Von bescheidenen Anfängen ist die E-Zigarettenindustrie auf ein geschätztes weltweites Marktvolumen von USD 2 Milliarden Ende 2012 gewachsen. Mit dem Markteintritt internationaler Tabakkonzerne in den E-Zigarettenmarkt ist es notwendig geworden, den regulatorischen Prozess vor Beeinflussung durch die Branche zu schützen (FCTC, Artikel 5.3).
6) Mehr Finanzmittel von internationalen und nationalen Regulierungsbehörden für die Untersuchung möglicher schädlicher versus nützlicher Auswirkungen von E-Zigaretten für den Einzelnen und die Gesellschaft.

Dies ist notwendig, damit Öffentlichkeit und Regulierungsbehörden wissenschaftlich fundiert entscheiden können, wie sie idealerweise mit E-Zigaretten umgehen wollen.

\section{Wieder den Wald statt lauter Bäumen sehen}

Es wird umfassend diskutiert, ob elektrische Zigaretten ein Segen sind oder eine zusätzliche Gefahr im Hinblick auf die weltweit führende vermeidbare Todesursache, den Tabakkonsum - ein Ende dieser Diskussionen ist nicht abzusehen. Unbestritten ist jedoch, dass die auf Verbrennung basierenden Tabakerzeugnisse wie Zigaretten, Zigarren, Pfeifen und Wasserpfeifen, die für den Löwenanteil der tabakbedingten Morbidität und Mortalität verantwortlich sind, selbst im günstigsten Fall nicht über Nacht verschwinden werden. Selbst in Best-Case-Szenarien geht die Branche davon aus, dass auch 2020 noch über $80 \%$ der Einnahmen der Tabakindustrie auf konventionelle Tabakrauchwaren entfallen werden und weniger als $20 \%$ auf Tabakprodukte mit modifiziertem Risiko. Wir sollten daher zwar darauf achten, in langfristigen Lösungsstrategien auch neuartige Tabakprodukte wie E-Zigaretten zu berücksichtigen, doch sollten wir unsere Bemühungen (und finanziellen und wissenschaftlichen Ressourcen) auch weiterhin primär darauf fokussieren, wie wir die globale Exposition gegenüber brennbaren Tabakprodukten reduzieren können schauen wir auf den großen Wald und nicht nur auf den einen exotischen Baum.

\section{Das Wichtigste in Kürze}

- Elektrische Zigaretten (E-Zigaretten) stellen neben anderen Nikotinabgabesystemen eine neue Klasse rauchfreier Tabakerzeugnisse dar, mit denen Nikotin inhaliert wird.

- Neben Nikotin in unterschiedlichen Mengen enthält das Liquid in E-Zigaretten Trägerflüssigkeit (Propylenglykol und/ oder Glycerin) und Aromastoffe (um die Attraktivität des Produkts zu erhöhen); einige Marken sind häufig durch giftige Verunreinigungen kontaminiert. Es sind derzeit über 7700 verschiedene Geschmacksrichtungen von mehr als 460 verschiedenen Marken auf dem Markt.

- Die Vor- und Nachteile von E-Zigaretten aus Sicht des Gesundheitswesens werden lebhaft diskutiert, sowohl in der Öffentlichkeit als auch in der Fachwelt.

- Da der Dampf von E-Zigaretten weniger Teer und krebserregende Stoffe enthält als Zigarettenrauch, könnten E-Zigaretten das Risiko von Erkrankungen verringern, die durch diese Stoffe verursacht werden.

- Zur Sicherheit der Geräte liegen jedoch erst wenige Daten vor. Es liegen Berichte über verschiedene potenzielle akut schädliche Auswirkungen auf die Gesundheit vor, Ergebnisse von Langzeitstudien fehlen jedoch noch. 
- Ebenso mangelt es - zumindest bisher - an wissenschaftlicher Evidenz dafür, dass E-Zigaretten zur Raucherentwöhnung beitragen können.

- Ein wichtiger Einwand ist die Möglichkeit, dass eine weitverbreitete Nutzung von E-Zigaretten auch das Rauchen wieder normalisieren und den Einstieg ins Rauchen fördern könnte, insbesondere bei jungen Menschen.

- Regulierungsmaßnahmen für E-Zigaretten müssen dringend ergriffen werden, einschließlich ihrer Regulierung als Tabakerzeugnisse.

\section{Zur Kenntnisnahme}

I.T.A. ist derzeit für das Office on Smoking and Health der Centers for Disease Control and Prevention tätig. Die diesem Bericht zugrunde liegende Forschung erfolgte außerhalb der offiziellen Aufgaben seiner aktuellen Position und spiegelt nicht die offizielle Politik oder Positionen der Centers for Disease Control and Prevention wider.

\section{Disclosure Statement}

Die Autoren haben keine Interessenkonflikte offenzulegen.

\section{Literatur}

1 e-cigarettes: a moral quandary. Lancet 2013; 382:914.

2 Lopez D, Collishaw NE, Piha T: A descriptive model of the cigarette epidemic in developed countries (editorial). Tob Control 1994;3:3.

3 Giovino GA: Epidemiology of tobacco use in the United States. Oncogene 2002;21:73267340 .

4 Benowitz NL: Emerging nicotine delivery products. Implications for public health. Ann Am Thorac Soc 2014;11:231-235.

5 Drummond MB, Upson D: Electronic cigarettes - potential harms and benefits. Ann Am Thorac Soc 2014;11:236-242.

6 Berridge V: Electronic cigarettes and history. Lancet 2014;383:2204-2205.

7 Hajek P, Goniewicz ML, Phillips A, Myers Smith K, West O, McRobbie H: Nicotine intake from electronic cigarettes on initial use and after 4 weeks of regular use. Nicotine Tob Res 2015;17:175-179.

8 Zhang Y, Sumner W, Chen DR: In vitro particle size distributions in electronic and conventional cigarette aerosols suggest comparable deposition patterns. Nicotine Tob Res 2013;15: 501-508.

9 Goniewicz ML, Kuma T, Gawron M, Knysak J, Kosmider L: Nicotine levels in electronic cigarettes. Nicotine Tob Res 2013;15:158-166.

10 Trtchounian A, Williams M, Talbot P: Conventional and electronic cigarettes (e-cigarettes) have different smoking characteristics. Nicotine Tob Res 2010;12:905-912.

11 Vansickel AR, Eissenberg T: Electronic cigarettes: effective nicotine delivery after acute administration. Nicotine Tob Res 2013;15:267270.

12 Schripp T, Markewitz D, Uhde E, Salthammer $\mathrm{T}$ : Does e-cigarette consumption cause passive vaping? Indoor Air 2013;23:25-31.

13 Uchiyama S, Ohta K, Inaba Y, Kunugita N: Determination of carbonyl compounds generated from the E-cigarette using coupled silica cartridges impregnated with hydroquinone and 2,4-dinitrophenylhydrazine, followed by highperformance liquid chromatography. Anal Sci 2013;29:1219-1222.

14 Kosmider L, Sobczak A, Fik M, Knysak J, Zaciera M, Kurek J, Goniewicz ML: Carbonyl compounds in electronic cigarette vapors: effects of nicotine solvent and battery output voltage. Nicotine Tob Res 2014;16:1319-1326.
15 Carpenter CM, Wayne GF, Connolly GN: The role of sensory perception in the development and targeting of tobacco products. Addiction 2007;102:136-147.

16 Farsalinos KE, Romagna G, Tsiapras D, Kyrzopoulos S, Spyrou A, Voudris V: Impact of flavour variability on electronic cigarette use experience: an internet survey. Int J Environ Res Public Health 2013; 10:7272-7282.

17 Trehy ML, Ye W, Hadwiger ME, Moore TW, Allgire JF, Woodruff JT, Ahadi SS, Black JC, Westenberger BJ: Analysis of electronic cigarette cartridges, refill solutions, and smoke for nicotine and nicotine related impurities. J Liq Chromatogr Relat Technol 2011;34: 1442-1458.

18 Goniewicz ML, Knysak J, Gawron M, Kosmider L, Sobczak A, Kurek J, Prokopowicz A, Jablonska-Czapla M, Rosik-Dulewska C, Havel C, Jacob P 3rd, Benowitz N: Levels of selected carcinogens and toxicants in vapour from electronic cigarettes. Tob Control 2014;23:133-139.

19 Wieslander G, Norback D, Lindgren T: Experimental exposure to propylene glycol mist in aviation emergency training: acute ocular and respiratory effects. Occup Environ Med 2001; 58:649-655.

20 Chen IL: FDA summary of adverse events on electronic cigarettes. Nicotine Tob Res 2013; 15:615-616.

21 Flouris AD, Chorti MS, Poulianiti KP, Jamurtas AZ, Kostikas K, Tzatzarakis MN, Wallace Hayes A, Tsatsakis AM, Koutedakis Y: Acute impact of active and passive cigarette smoking on serum cotinine and lung function. Inhal Toxicol 2013;25:91-101.

22 Vardavas CI, Anagnostopoulos N, Kougias M, Evangelopoulou V, Connolly GN, Behrakis PK: Short-term pulmonary effects of using an electronic cigarette: impact on respiratory flow resistance, impedance, and exhaled nitric oxide. Chest 2012;141:1400-1406.

23 Vakali S, Tsikrika S, Gennimata S, Kaltsakas G, Palamidas A, Koulouris A, Gratziou C: E-Cigarette acute effect on symptoms and airway inflammation: comparison of nicotine with a non-nicotine cigarette. Tob Induc Dis 2014;12 (suppl 1):A35.

24 Tsikrika S, Vakali S, Gennimata S, Palamidas A, Kaltsakas G, Koulouris N, Gratziou C: Short term use of an e-cig: influence on clinical symptoms, vital signs and eCO levels. Tob Induc Dis 2014;12(suppl 1):A30.
25 Lim HB, Kim SH: Inhalation of e-cigarette cartridge solution aggravates allergen-induced airway inflammation and hyper-responsiveness in mice. Toxicol Res 2014;30:13-18.

26 Flouris AD, Poulianiti KP, Chorti MS, Jamurtas AZ, Kouretas D, Owolabi EO, Tzatzarakis MN, Tsatsakis AM, Koutedakis Y: Acute effects of electronic and tobacco cigarette smoking on complete blood count. Food Chem Toxicol 2012; 50:3600-3603.

27 Bahl V, Lin S, Xu N, Davis B, Wang YH, Talbot $\mathrm{P}$ : Comparison of electronic cigarette refill fluid cytotoxicity using embryonic and adult models. Reprod Toxicol 2012;34:529-537.

28 Romagna G, Allifranchini E, Bocchietto E, Todeschi S, Esposito M, Farsalinos KE: Cytotoxicity evaluation of electronic cigarette vapor extract on cultured mammalian fibroblasts (ClearStream-LIFE): comparison with tobacco cigarette smoke extract. Inhal Toxicol 2013;25: 354-361.

29 Farsalinos KE, Romagna G, Allifranchini E, Ripamonti E, Bocchietto E, Todeschi S, Tsiapras D, Kyrzopoulos S, Voudris V: Comparison of the cytotoxic potential of cigarette smoke and electronic cigarette vapour extract on cultured myocardial cells. Int J Environ Res Public Health 2013;10:5146-5162.

30 Zhu SH, Sun JY, Bonnevie E, Cummins SE, Gamst A, Yin L, Lee M: Four hundred and sixty brands of e-cigarettes and counting: implications for product regulation. Tob Control 2014;23(suppl 3):iii3-iii9.

31 Williams M, Villarreal A, Bozhilov K, Lin S, Talbot P: Metal and silicate particles including nanoparticles are present in electronic cigarette cartomizer fluid and aerosol. PLoS One 2013;8:e57987.

32 Czogala J, Goniewicz ML, Fidelus B, ZielinskaDanch W, Travers MJ, Sobczak A: Secondhand exposure to vapors from electronic cigarettes. Nicotine Tob Res 2014; 16:655-662.

33 Hutzler C, Paschke M, Kruschinski S, Henkler F, Hahn J, Luch A: Chemical hazards present in liquids and vapors of electronic cigarettes. Arch Toxicol 2014;88:1295-1308.

34 Bjartveit K, Tverdal A: Health consequences of smoking 1-4 cigarettes per day. Tob Control 2005;14:315-320.

35 Frost-Pineda K, Appleton S, Fisher M, Fox K, Gaworski CL: Does dual use jeopardize the potential role of smokeless tobacco in harm reduction? Nicotine Tob Res 2010;12:1055-1067. 
36 Caponnetto P, Campagna D, Cibella F, Morjaria JB, Caruso M, Russo C, Polosa R: EffiCiency and Safety of an eLectronic cigAreTte (ECLAT) as tobacco cigarettes substitute: a prospective 12-month randomized control design study. PLoS One 2013;8:e66317.

37 Orr KK, Asal NJ: Efficacy of electronic cigarettes for smoking cessation. Ann Pharmacother 2014;48:1502-1506.

38 Bullen C, Howe C, Laugesen M, McRobbie H, Parag V, Williman J, Walker N: Electronic cigarettes for smoking cessation: a randomised controlled trial. Lancet 2013;382:1629-1637.

39 Brown J, Beard E, Kotz D, Michie S, West R: Real-world effectiveness of e-cigarettes when used to aid smoking cessation: a cross-sectional population study. Addiction 2014;109:15311540 .

40 Vardavas CI, Filippidis FT, Agaku IT: Determinants and prevalence of e-cigarette use throughout the European Union: a secondary analysis of 26,566 youth and adults from 27 countries. Tob Control 2015;24:442-448.

41 Grana R, Benowitz N, Glantz SA: E-cigarettes: a scientific review. Circulation 2014;129:19721986.

42 IOM (Institute of Medicine): Scientific Standards for Studies on Modified Risk Tobacco Products. Washington, The National Academies Press, 2012.

43 Pokhrel P, Fagan P, Little MA, Kawamoto CT, Herzog TA: Smokers who try e-cigarettes to quit smoking: findings from a multiethnic study in Hawaii. Am J Public Health 2013; 103:e57-e62.

44 Choi K, Forster JL: Beliefs and experimentation with electronic cigarettes: a prospective analysis among young adults. Am J Prev Med 2014;46:175-178.

45 Dockrell M, Morrison R, Bauld L, McNeill A: E-cigarettes: prevalence and attitudes in Great Britain. Nicotine Tob Res 2013;15:1737-1744.

46 Kandel ER, Kandel DB: Shattuck Lecture. A molecular basis for nicotine as a gateway drug. N Engl J Med 2014;371:932-943.

47 Bunnell RE, Agaku IT, Arrazola RA, Apelberg BJ, Caraballo RS, Corey CG, Coleman BN, Dube SR, King BA: Intentions to smoke cigarettes among never-smoking U.S. middle and high school electronic cigarette users, National Youth Tobacco Survey, 2011-2013. Nicotine Tob Res 2015; 17:228-235.

48 Pearson JL, Richardson A, Niaura RS, Vallone DM, Abrams DB: e-cigarette awareness, use, and harm perceptions in US adults. Am J Public Health 2012;102:1758-1766.

49 Regan AK, Promoff G, Dube SR, Arrazola R: Electronic nicotine delivery systems: adult use and awareness of the 'e-cigarette' in the USA. Tob Control 2013;22:19-23.
50 Dutra LM, Glantz SA: Electronic cigarettes and conventional cigarette use among U.S. adolescents: a cross-sectional study. JAMA Pediatr 2014;168:610-617.

51 King BA, Alam S, Promoff G, Arrazola R, Dube SR: Awareness and ever-use of electronic cigarettes among U.S. adults, 2010-2011. Nicotine Tob Res 2013;15:1623-1627.

52 Lee S, Grana RA, Glantz SA: Electronic cigarette use among Korean adolescents: a crosssectional study of market penetration, dual use, and relationship to quit attempts and former smoking. J Adolesc Health 2014;54:684690

53 Frieden T: E-Cigarette Use More than Doubles among U.S. Middle and High School Students from 2011-2012. Atlanta, Centers for Disease Control and Prevention, 2013, http://www.cdc. gov/media/releases/2013/p0905-ecigarette-use. html.

54 Blasi F, Ward B: Electronic nicotine delivery systems (ENDS): the beginning of the end or the end of the beginning? Eur Respir J 2014;44: 585-588.

55 King AC, Smith LJ, McNamara PJ, Matthews AK, Fridberg DJ: Passive exposure to electronic cigarette (e-cigarette) use increases desire for combustible and e-cigarettes in young adult smokers. Tob Control 2015;24:501-504.

56 Vakkalanka JP, Hardison LS Jr, Holstege CP: Epidemiological trends in electronic cigarette exposures reported to U.S. Poison Centers. Clin Toxicol (Phila) 2014;52:542-548.

57 Chatham-Stephens K, Law R, Taylor E, Melstrom P, Bunnell R, Wang B, Apelberg B, Schier JG; Centers for Disease Control and Prevention (CDC): Notes from the field: calls to poison centers for exposures to electronic cigarettes United States, September 2010 - February 2014. MMWR Morb Mortal Wkly Rep 2014; 63:292-293.

58 US Department of Health and Human Services: The Health Consequences of Smoking - 50 Years of Progress: A Report of the Surgeon General. Atlanta, US Department of Health and Human Services, Centers for Disease Control and Prevention, National Center for Chronic Disease Prevention and Health Promotion, Office on Smoking and Health, 2014.

59 Schober W, Szendrei K, Matzen W, OsianderFuchs H, Heitmann D, Schettgen T, Jorres RA, Fromme H: Use of electronic cigarettes (e-cigarettes) impairs indoor air quality and increases FeNO levels of e-cigarette consumers. Int J Hyg Environ Health 2014;217:628-637.

60 Ballbè M, Martínez-Sánchez JM, Sureda X, Fu M, Pérez-Ortuño R, Pascual JA, Saltó E, Fernández E: Cigarettes vs e-cigarettes: passive exposure at home measured by means of airborne marker and biomarkers. Environ Res 2014;135C:76-80.
61 Goniewicz ML, Lee L: Electronic cigarettes are a source of thirdhand exposure to nicotine. Nicotine Tob Res 2015;17:256-258.

62 Schraufnagel DE, Blasi F, Drummond MB, Lam DC, Latif E, Rosen MJ, Sansores R, Van Zyl-Smit R; Forum of International Respiratory Societies: Electronic cigarettes. A position statement of the Forum of International Respiratory Societies. Am J Respir Crit Care Med 2014;190:611-618.

63 Bhatnagar A, Whitsel LP, Ribisl KM, Bullen C, Chaloupka F, Piano MR, Robertson RM, McAuley T, Goff D, Benowitz N; American Heart Association Advocacy Coordinating Committee, Council on Cardiovascular and Stroke Nursing, Council on Clinical Cardiology, Council on Quality of Care and Outcomes Research: Electronic cigarettes: a policy statement from the American Heart Association. Circulation 2014;130:1418-1436.

64 Brandon TH, Goniewicz ML, Hanna NH, Hatsukami DK, Herbst RS, Hobin JA, Ostroff JS, Shields PG, Toll BA, Tyne CA, Viswanath K, Warren GW: Electronic nicotine delivery systems: a policy statement from the American Association for Cancer Research and the American Society of Clinical Oncology. Clin Cancer Res 2015;21:514-525.

65 WHO Framework Convention on Tobacco Control: Sixth Session. Moscow, Russian Federation, 13-18 October 2014. Decision: Electronic Nicotine Delivery Systems and Electronic Non-Nicotine Delivery Systems. http://apps. who.int/gb/fctc/PDF/cop6/FCTC_COP6(9)-en. $p d f$

66 Grana RA, Ling PM: 'Smoking revolution': a content analysis of electronic cigarette retail websites. Am J Prev Med 2014;46:395-403.

67 Directive 2014/40/EU of the European Parliament and of the Council of 3 April 2014 on the approximation of the laws, regulations and administrative provisions of the Member States concerning the manufacture, presentation and sale of tobacco and related products and repealing Directive 2001/37/EC Text with EEA relevance. http://eur-lex.europa.eu/legal-content/ EN/TXT/?qid=1398761379066ぬuri=OJ:JOL_2 014_127_R_0001.

68 Orleans CT, Slade J: Nicotine Addiction: Principles and Management. New York, Oxford University Press, 2002.

69 Bertholon JF, Becquemin MH, Annesi-Maesano I, Dautzenberg B: Electronic cigarettes: a short review. Respiration 2013;86:433-438. 